\title{
APPLICATION OF MEASURE OF NONCOMPACTNESS TO VOLTERRA EQUATIONS OF CONVOLUTION TYPE
}

\author{
EDGARDO ALVAREZ AND CARLOS LIZAMA
}

Communicated by Juan J. Trujillo

\begin{abstract}
Sufficient conditions for the existence of at least one solution of a nonlinear integral equation with a general kernel are established. The existence result is proved in $C([0, T], E)$, where $E$ denotes an arbitrary Banach space. We use the Darbo-Sadovskii fixed point theorem and techniques of measure of noncompactness. We extend and generalize results obtained by other authors in the context of fractional differential equations. One example illustrates the theoretical results.
\end{abstract}

1. Introduction. During the past few years the theory of functional Volterra integral equations has undergone rapid development. The growth has been strongly promoted by the large number of applications that this theory has found in rheology, risk theory, renewal theory, branching processes, queuing theory and inventory analysis [12]. Recent developments include studies on state dependent delay [16], perturbations [13] and attractive behavior of solutions [6, 7].

In this paper, we study existence of solutions for the following nonlinear Volterra integral equation of convolution type:

$$
u(t)=\int_{0}^{t} a(t-s) f(s, u(s)) d s \quad t \in[0, T],
$$

where $a \in L_{\text {loc }}^{1}\left(\mathbb{R}_{+}\right)$is a scalar kernel, $E$ is a Banach space with the norm $\|\cdot\|$ and $f:[0, T] \times E \rightarrow E$ satisfies some suitable conditions.

2010 AMS Mathematics subject classification. Primary 34A12, 45D05, 45N05.

Keywords and phrases. Volterra equations of convolution type, nonlinear functional integral equations, Darbo's fixed point theorem, measure of noncompactness.

The first author is partially supported by Dirección de Investigaciones Universidad del Norte, project No. 2016-011. The second author is partially supported by CONICYT, under Fondecyt grant No. 1140258.

Received by the editors on January 28, 2016. 
Equation (1.1) was studied by Aghajani, Pourhadi and Trujillo [3] for the case

$$
a(t)=\frac{t^{\alpha-1}}{\Gamma(\alpha)} \quad \text { and } \quad 0<\alpha<1 .
$$

See [3, Lemma 3.1] and [21] where the precise notion of solution is defined. They investigated the existence of solutions for the associated Cauchy problem using a nice generalization of Darbo's fixed point theorem via the Hausdorff measure of noncompactness and gave interesting concrete examples.

The technique of measure of noncompactness for solving evolution equations has been used increasingly in the past years due to its efficiency when compared with other methods. For instance, Banaś and Nalepa showed [10] that, with the help of that measure of noncompactness, one can obtain an existence result for a nonlinear quadratic integral equation of Hammerstein type in Hölder spaces. Existence results for impulsive differential equations with nonlocal conditions via measures of noncompactness has been proved by Mallika Arjunan, Kavitha and Selvi [18]. Also, Allahyari, Arab and Shole Haghighi studied [5] the existence of solutions for some classes of integro-differential equations. However, this study does not include the convolution type equation (1.1). Nonlinear functional equations of convolution type on the line and with external force term has been studied recently in Lebesgue spaces by Khosravi, Allahyari and Shole Haghighi [15]. On the other hand, application of the measure of noncompactness to $\ell_{1}$-solvability of infinite systems of second order differential equations has been considered by Aghajani and Pourhadi [2] and by Mursaleen and Rizvi [20]. Also, the authors [19] studied an application of measure of noncompactness to $\ell_{p}$-solvability of infinite systems of differential equations. For an up-to-date account of this method and its applications, also see the recent monograph by Banaś and Mursaleen [9].

Since fractional differential equations are particular cases of functional integral equations of the type (1.1), it is natural to ask, for the following problem:

(Q) For which class of kernels $a(t)$ does there exist a solution for $(1.1)$ ?

We remark that this is an inverse problem in terms of the kernel $a(t)$, and therefore, it is not included in the recent reference [14] where 
existence of solutions for a very general class of integral equations was studied.

We have success in solving problem (Q) for the class of kernels $a(t)$ that satisfy the following condition: there exists $0<\sigma<1$ such that $a \in L^{1 / \sigma}([0, T] ; \mathbb{R})$ and

$$
\lim _{h \rightarrow 0} \int_{0}^{T}|a(s+h)-a(s)|^{1 /(1-\sigma)} d s=0
$$

holds.

It is interesting to note that the class of kernels defined by $a(t)=$ $e^{-\delta t}$ satisfy our assumptions under some mild restrictions on the parameter $\delta>0$. This class of kernel naturally appears in the theory of integral equations of convolution type. It corresponds to Maxwell type materials in viscoelasticity theory (relaxation modulus). See $[\mathbf{1 7}$, Chapter 2, subsection 2.4]. A concrete example is provided in the last section of this paper.

In order to obtain our results, we use the properties of the measure of noncompactness and a consequence of the Darbo-Sadovskii fixed point theorem (see $[\mathbf{1}, \mathbf{2}])$ to prove the existence of solutions of equation (1.1) under the hypothesis that the nonlinearity $f(t, u)$ satisfies the Carathéodory type condition, and it is dominated by a control function which is radial with respect to $u$.

The outline of this paper is as follows. Section 2 is devoted to preliminaries, recalling the definition and fundamental properties of measure of noncompactness, mentioning a generalization of Darbo fixed point theorem due to Aghajani, Banaś and Sabzali [1, Theorem 2.2], and recalling Bothe's lemma (see [11]) which is the essential ingredient in the proof of our main result.

Section 3 shows the existence of at least one solution of (1.1) on an arbitrary Banach space $E$ by using arguments of the measure of noncompactness, a consequence of Darbo-Sadovskii fixed point theorem and, again, certain hypotheses on the kernel and the function $f$.

In Section 4, an application is provided which indicates how our theorems can be applied to more concrete problems. 
In Section 5, we give a concrete example of our main result considering as external nonlinear force:

$$
f(t, x)=\frac{1}{t^{2}+(1 / 2)}\left(\ln \left(\left|x_{k}\right|+1\right)+\frac{t}{k^{2}}\right)_{k=1}^{\infty},
$$

for every $x=\left(x_{k}\right) \in c_{0}$, where $c_{0}$ is the Banach space of null sequences which consists of all sequences whose limit is zero, as well as the Maxwell type materials present in the kernel $a(t)=e^{-\delta t}$.

Finally, it is interesting to observe that, for this function $f$, the existence of at least one solution on $c_{0}$ is ensured under the hypothesis $0<\delta<2$ and $0<T<1 / 2$.

2. Preliminaries. Suppose that $(E,\|\cdot\|)$ is a Banach space and denote by $C([0, T], E)$ the space of all continuous functions equipped with the norm

$$
\|u\|_{\infty}=\sup _{s \in[0, T]}\|u(s)\| .
$$

Let $L^{1}([0, T] ; E)$ be the space of all Bochner integrable functions $h:[0, T] \rightarrow E$ endowed with the norm

$$
\|h\|_{L^{1}([0, T] ; E)}=\int_{0}^{T}\|h(s)\|_{E} d s .
$$

Now, we mention some results and facts about measure of noncompactness.

We denote by $\bar{X}$ and Conv $X$ the closure and the convex closure of $X$ as a subset of $E$, respectively. Further, we denote by $\mathfrak{M}_{E}$ the family of all nonempty bounded subsets of $E$ and $\mathfrak{R}_{E}$ its subfamily consisting of all relatively compact sets.

We recall that the Hausdorff of measure of noncompactness $\gamma$ defined on a bounded set $M$ of a Banach space $E$ is given by:

$$
\gamma(B)=\inf \{\epsilon>0: B \text { has a finite cover by balls of radius } \epsilon\} .
$$

Definition 2.1 ([8]). A mapping $\gamma: \mathfrak{M}_{E} \rightarrow \mathbb{R}^{+}$is said to be a measure of noncompactness in $E$ if satisfies the following conditions:

(a) the family $\operatorname{ker} \gamma=\left\{X \in \mathfrak{M}_{E}: \gamma(X)=0\right\}$ is nonempty and $\operatorname{ker} \gamma \subset \mathfrak{R}_{E}$, 
(b) $X \subset Y$ implies $\gamma(X) \leq \gamma(Y)$,

(c) $\gamma(\bar{X})=\gamma(X)$,

(d) $\gamma(\operatorname{Conv}(X))=\gamma(X)$,

(e) $\gamma(X \cup Y)=\max \{\gamma(X), \gamma(Y)\}$,

(f) $\gamma(r X)=|r| \gamma(X)$ for any $r \in \mathbb{R}$,

(g) $\gamma(X+Y) \leq \gamma(X)+\gamma(Y)$.

(h) If the mapping $T: D(T) \subset E \rightarrow F$ is Lipschitz continuous with constant $k$, then $\gamma_{F}(T B) \leq k \gamma_{E}(B)$ for any bounded set $B \subset D(T)$, where $F$ is a Banach space and $\gamma_{A}$ denotes the measure of noncompactness corresponding to a Banach space $A$.

(i) For all $\lambda \in[0,1]$,

$$
\gamma(\lambda X+(1-\lambda) Y) \leq \lambda \gamma(X)+(1-\lambda) \gamma(Y) .
$$

(j) If $\left(X_{n}\right)_{n \in \mathbb{N}}$ is a sequence of closed sets from $\mathfrak{M}_{E}$ such that

$$
X_{n+1} \subset X_{n} \text { for all } n=1,2,3 \ldots \text {, and } \lim _{n \rightarrow \infty} \gamma\left(X_{n}\right)=0 \text {, }
$$

then

$$
X_{\infty}=\bigcap_{n=1}^{\infty} X_{n} \neq \emptyset .
$$

The family ker $\gamma$ described in (a) is said to be the kernel of the measure of noncompactness $\gamma$.

Let $I:=[0, T]$. We need the following results.

Lemma $2.2([8])$. If $W \subset C(I, E)$ is bounded and equicontinuous, then the set $\gamma(W(t))$ is continuous on $I$ and

$$
\begin{aligned}
\gamma(W) & =\sup _{t \in I} \gamma(W(t)), \\
\gamma\left(\int_{0}^{t} W(s) d s\right) & \leq \int_{0}^{t} \gamma(W(s)) d s .
\end{aligned}
$$

Lemma 2.3 ([11]). If $\left(u_{n}\right)_{n \in \mathbb{N}} \subset L^{1}(I ; E)$ satisfies $\left\|u_{n}\right\| \leq \kappa(t)$ almost everywhere on $I$ for all $n \geq 1$ and for some $\kappa \in L^{1}(I ;(0, \infty))$, then the function $\gamma\left(\left(u_{n}(t)\right)_{n \in \mathbb{N}}\right)$ belongs to $L^{1}(I ;(0, \infty))$ and

$$
\gamma\left(\left\{\int_{0}^{t} u_{n}(s) d s: n \geq 1\right\}\right) \leq 2 \int_{0}^{t} \gamma\left(\left\{u_{n}(s): n \geq 1\right\} d s .\right.
$$


The following result will be the key tool in the proof of our main result.

Lemma 2.4 ([11]). If $W \subset C(I, E)$ is bounded, then, for each $\epsilon>0$, there exists a sequence $\left(u_{n}\right)_{n \in \mathbb{N}} \subset W$ such that

$$
\gamma(W) \leq 2 \gamma\left(\left(u_{n}\right)_{n \in \mathbb{N}}\right)+\epsilon .
$$

To prove our main result, we need the following theorem due to $[\mathbf{1}$, Theorem 2.2], which is a generalization of Darbo's fixed point.

Theorem 2.5 ([1, Theorem 2.2]). Let $C$ be a nonempty, bounded, closed and convex subset of a Banach space $E$, and let $T: C \rightarrow C$ be a continuous function satisfying

$$
\gamma(T(W)) \leq \phi(\gamma(W))
$$

for each $W \subset C$, where $\gamma$ is an arbitrary measure of noncompactness and $\phi: \mathbb{R}^{+} \rightarrow \mathbb{R}^{+}$is a monotone increasing (not necessarily continuous) function with

$$
\lim _{n \rightarrow \infty} \phi^{n}(t)=0 \quad \text { for all } t \geq 0 .
$$

Then $T$ has at least one fixed point in $C$.

3. Existence of solutions. In this section, we investigate solutions of the problem:

$$
u(t)=\int_{0}^{t} a(t-s) f(s, u(s)) d s, \quad t \in[0, T],
$$

where $a \in L_{\text {loc }}^{1}\left(\mathbb{R}_{+}\right)$is a scalar kernel, $E$ is a Banach space with the norm $\|\cdot\|$ and $f:[0, T] \times E \rightarrow E$ satisfies some suitable conditions.

We need the following assumptions:

(H1) $f$ satisfies Carathéodory type conditions, i.e., $f(\cdot, x)$ is measurable for each $x$ and $f(t, \cdot)$ is continuous for almost every $t \in I$. 
(H2) For any $0<q<1$, there exists a function $m \in L^{1 / \beta}\left(I ; \mathbb{R}^{+}\right)$ where $0<\beta<q$ and a nondecreasing continuous function $\Phi: \mathbb{R}^{+} \rightarrow \mathbb{R}^{+}$such that

$$
\|f(t, x)\| \leqslant m(t) \Phi(\|x\|),
$$

for all $x \in E$ and almost all $t \in I$.

(H3) There exists a function $H \in L^{1}\left(I ; \mathbb{R}^{+}\right)$such that, for any bounded $B \subseteq X$,

$$
\gamma(f(t, B)) \leqslant H(t) \phi(\gamma(B))
$$

for almost all $t \in I$. (Here $\phi$ is some function that satisfies the same conditions of Theorem 2.5.)

(H4) $a \in L^{1 / \beta}(I ; \mathbb{R})$ and satisfies

$$
\lim _{h \rightarrow 0} \int_{0}^{T}|a(\tau+h)-a(\tau)|^{1 /(1-\beta)} d \tau=0 .
$$

(H5) There exists at least one solution $p \in C(I,(0, \infty))$ to the inequality

$$
\Phi\left(\|p\|_{0}\right) \int_{0}^{t} a(t-s) m(s) d s \leq p(t), \quad t \in I,
$$

where $\|\cdot\|_{0}$ is the sup norm in $C(I,(0, \infty))$.

The next theorem is the main result of this paper.

Theorem 3.1. Suppose that the hypotheses (H1)-(H5) hold. Then problem (3.1) has at least one solution $u \in C(I, E)$.

Proof. Let $\mathcal{F}: C(I, E) \rightarrow C(I, E)$ be defined by

$$
(\mathcal{F} u)(t):=\int_{0}^{t} a(t-s) f(s, u(s)) d s, \quad t \in I,
$$

for all $u \in C(I, E)$. Let $p(t)$ be the function which satisfies (H5) and $p_{0}=\inf _{t \in I} p(t)>0$. We define

$$
B_{p}:=\left\{u \in C(I, E):\|u\|_{E} \leq p_{0}\right\} .
$$

Set

$$
C_{p}:=\operatorname{Conv} \mathcal{F} B_{p}
$$


We proceed to verify the hypothesis of Theorem 2.5, dividing the proof into five steps.

Step 1. Conv $\mathcal{F} B_{p}$ is equicontinuous. It is enough to prove that $\mathcal{F} B_{p}$ is equicontinuous because the concepts of boundedness and equicontinuity have hereditary properties related to the closure of the convex hull in $C(I, E)$.

Indeed, let $u \in B_{p}$, and let $0 \leq t_{1}<t_{2} \leq T$ be given. Then

$$
\begin{aligned}
&\left\|\mathcal{F}(u)\left(t_{2}\right)-\mathcal{F}(u)\left(t_{1}\right)\right\| \\
& \leq\left\|\int_{0}^{t_{2}} a\left(t_{2}-s\right) f(s, u(s)) d s-\int_{0}^{t_{1}} a\left(t_{1}-s\right) f(s, u(s)) d s\right\| \\
& \leq \int_{0}^{t_{1}}\left|a\left(t_{2}-s\right)-a\left(t_{1}-s\right)\right|\|f(s, u(s))\| d s \\
&+\int_{t_{1}}^{t_{2}}\left|a\left(t_{2}-s\right)\right|\|f(s, u(s))\| d s \\
& \leq \int_{0}^{t_{1}}\left|a\left(t_{2}-s\right)-a\left(t_{1}-s\right)\right| m(s) \Phi(\|u(s)\|) d s \\
&+\int_{t_{1}}^{t_{2}}\left|a\left(t_{2}-s\right)\right| m(s) \Phi(\|u(s)\|) d s \\
& \leq \int_{0}^{t_{1}}\left|a\left(t_{2}-s\right)-a\left(t_{1}-s\right)\right| m(s) \Phi(\|p(s)\|) d s \\
&+\int_{t_{1}}^{t_{2}}\left|a\left(t_{2}-s\right)\right| m(s) \Phi(\|p(s)\|) d s \\
& \leq \Phi\left(\|p\|_{0}\right)\left(\int_{0}^{t_{1}}\left|a\left(t_{2}-s\right)-a\left(t_{1}-s\right)\right| m(s) d s\right.
\end{aligned}
$$

It follows from the Hölder inequality and (H4) that

$$
\begin{aligned}
& \left\|\mathcal{F}(u)\left(t_{2}\right)-\mathcal{F}(u)\left(t_{1}\right)\right\| \\
& \quad \leq \Phi\left(\|p\|_{0}\right)\|m\|_{L^{1 / \beta}(I,(0, \infty))} \\
& \quad \times\left[\left(\int_{0}^{T}\left|a\left(\left(t_{2}-t_{1}\right)+\tau\right)-a(\tau)\right|^{1 /(1-\beta)} d \tau\right)^{1-\beta}\right.
\end{aligned}
$$




$$
\left.+\left(\int_{0}^{t_{2}-t_{1}}|a(s)|^{1 / \beta} d s\right)^{\beta}\right] \longrightarrow 0 \quad \text { as } t_{1} \rightarrow t_{2}
$$

Step 2. $\mathcal{F}$ is continuous on $C(I, E)$. Since $f$ satisfies conditions (H1) and (H2), then $\mathcal{F}$ is well defined (consequence of Young's inequality).

Let $\left(u_{n}\right)$ be a sequence of functions in $C(I, E)$ which converges to $u \in C(I, E)$. We need to prove that $\left\|\mathcal{F} u_{n}-\mathcal{F} u\right\|_{\infty} \rightarrow 0$ as $n \rightarrow \infty$. Since $f(t, \cdot)$ is continuous, then $\left\|f\left(s, u_{n}(s)\right)-f(s, u(s))\right\| \rightarrow 0$ as $n \rightarrow \infty$. On the other hand, it follows from condition (H2) that

$$
\left\|f\left(s, u_{n}(s)\right)-f(s, u(s))\right\| \leq 2 m(s)\left[\Phi\left(\left\|u_{n}(s)\right\|\right)+\Phi(\|u(s)\|)\right] .
$$

By (H2) and (H4), we have that $a(t)$ and $m(t)$ are integrable. Therefore, the inequality:

$$
\begin{aligned}
& \int_{0}^{t} \mid a(t-s) \| m(s)|| \Phi(\|u(s)\|) \mid d s \\
& \leq \int_{0}^{t}|a(t-s) \| m(s)|\left|\Phi\left(\|u\|_{\infty}\right)\right| d s \\
& \quad=\Phi\left(\|u\|_{\infty}\right) \int_{0}^{t}|a(t-s)||m(s)| d s
\end{aligned}
$$

yields that $s \mapsto a(t-s) m(s) \Phi(\|u(s)\|)$ is also Lebesgue integrable on $[0, T]$. By the dominated convergence theorem, we obtain

$$
\begin{aligned}
& \left\|\left(\mathcal{F} u_{n}\right)(t)-(\mathcal{F}) u(t)\right\| \\
& \quad \leq \int_{0}^{t} \mid a(t-s)\left\|f\left(s, u_{n}(s)\right)-f(s, u(s))\right\| d s \longrightarrow 0 \quad \text { as } n \rightarrow \infty
\end{aligned}
$$

for all $t \in I$. Hence, $\left\|\mathcal{F} u_{n}-\mathcal{F} u\right\|_{\infty} \rightarrow 0$ on $I$ as $n \rightarrow \infty$. We conclude that $\mathcal{F}$ is continuous on $C(I, E)$.

Step 3. $\mathcal{F}$ is bounded from $B_{p}$ into itself. Let $u \in B_{p}$ and $t \in I$. Using (H2) and (H5), we get

$$
\begin{aligned}
\|\mathcal{F} u(t)\| & \leq \int_{0}^{t}|a(t-s)|\|f(s, u(s))\| d s \\
& \leq \int_{0}^{t} \mid a(t-s) m(s) \Phi(\|u(s)\|) d s
\end{aligned}
$$




$$
\begin{aligned}
& \leq \int_{0}^{t} \mid a(t-s) m(s) \Phi(\|p(s)\|) d s \\
& \leq \Phi\left(\|p\|_{0}\right) \int_{0}^{t} \mid a(t-s) m(s) d s \leq p(t), \quad t \in I .
\end{aligned}
$$

The conclusion follows. This step also proves that $\mathcal{F}\left(B_{p}\right) \subset B_{p}$.

Step 4. $\mathcal{F}: C_{p} \rightarrow C_{p}$ is well-defined and continuous. Note that $B_{p} \subset C(I, E)$ is bounded, closed and convex. Since $\mathcal{F}\left(B_{p}\right) \subset B_{p}$ from the preceding step and from the fact that $B_{p}$ is convex, we obtain

$$
C_{p}=\operatorname{Conv} \mathcal{F} B_{p} \subset \operatorname{Conv} B_{p}=B_{p} .
$$

Since the convex hull of a subset is the smallest convex set that contains to the subset, we have that:

$$
\mathcal{F} C_{p} \subset \mathcal{F} B_{p} \subset \operatorname{Conv} \mathcal{F} B_{p}=C_{p} .
$$

This shows that $\mathcal{F}: C_{p} \rightarrow C_{p}$ is well defined. From Step 2, we have that $\mathcal{F}$ is continuous on $C_{p}$.

Step 5. Let $C \subset C_{p}$ be arbitrary. By Step 4, we have that $C$ is bounded. From Lemma 2.4, we infer that, given $\epsilon>0$, there exists a sequence $\left(u_{n}\right)_{n \in \mathbb{N}} \subset C$ such that

$$
\begin{aligned}
\gamma(\mathcal{F} C)(t)) & =\gamma\left(\left\{\int_{0}^{t} a(t-s) f(s, u(s)) d s: u \in C\right\}\right) \\
& \leq 2 \gamma\left(\left\{\int_{0}^{t} a(t-s) f\left(s,\left(u_{n}(s)\right)_{n \in \mathbb{N}}\right) d s: u \in C\right\}\right)+\epsilon .
\end{aligned}
$$

Next, Lemma 2.2 and Lemma 2.3 together with condition (H3) imply

$$
\begin{aligned}
\gamma(\mathcal{F} C)(t)) & \leq 4 \int_{0}^{t} a(t-s) \gamma\left(\left\{f\left(s, u_{n}(s)\right): n \in \mathbb{N}\right\}\right) d s+\epsilon \\
& \leq 4 \int_{0}^{t} a(t-s) H(s) \gamma\left(\phi\left(\gamma\left(\left(u_{n}\right)_{n \in \mathbb{N}}\right)\right)\right) d s+\epsilon \\
& \leq 4 \phi\left(\gamma\left(\left(u_{n}\right)_{n \in \mathbb{N}}\right)\right) \int_{0}^{t} a(t-s) H(s) d s+\epsilon \\
& \leq 4 \phi\left(\gamma\left(\left(u_{n}\right)_{n \in \mathbb{N}}\right)\right) \int_{0}^{T} a(t-s) H(s) d s+\epsilon \\
& \leq 4 \phi(\gamma(C))(a * H)(T)+\epsilon
\end{aligned}
$$


where we have used in the last inequality that $\phi$ is increasing and the fact that $(a * H)(t)$ is well defined. Since $\epsilon>0$ is arbitrary, we have

$$
\gamma(\mathcal{F} C)(t)) \leq 4(a * H)(T) \phi(\gamma(C))
$$

Let

$$
\psi(s)=4(a * H)(T) \phi(s) .
$$

It follows from the properties of $\phi$ that $\psi:[0, \infty) \rightarrow[0, \infty)$ is monotone increasing and $\lim _{n \rightarrow \infty} \psi^{n}(t)=0$ for all $t \geq 0$. Also, by (3.4), we obtain that $\gamma(\mathcal{F} C)(t)) \leq \psi(\gamma(C))$.

We have proved that $\mathcal{F}: C_{p} \rightarrow C_{p}$ is a continuous function satisfying

$$
\gamma(\mathcal{F} C)(t)) \leq \psi(\gamma(C))
$$

for each $C \subset C_{p}$, where $\gamma$ is a measure of noncompactness and $\psi$ : $[0, \infty) \rightarrow[0, \infty)$ is a monotone increasing function with $\lim _{n \rightarrow \infty} \psi^{n}(t)=$ 0 for all $t \geq 0$. If follows from Theorem 2.5 that $\mathcal{F}$ has at least one fixed point $u \in C_{p} \subset C(I, E)$.

Remark 3.2. We observe that condition (H4) fails in the case of $a(s)=s^{q}$ for $q<-\beta$.

As a particular case, we recover the main result on the existence of solutions to the Cauchy problem for the fractional difference equation $D_{t}^{\alpha} u(t)=f(t, u(t)), 0<\alpha<1$, established by Aghajani, Pourhadi and Trujillo [3, Theorem 3.1].

Theorem 3.3. Consider the equation

$$
u(t)=\frac{1}{\Gamma(q)} \int_{0}^{t}(t-s)^{q-1} f(s, u(s)) d s, \quad t \in[0, T],
$$

where $q \in(0,1), E$ is a Banach space with the norm $\|\cdot\|$ and $f:[0, T] \times E \rightarrow E$ satisfies conditions $(\mathrm{H} 1),(\mathrm{H} 3)$ and

$(\mathrm{H} 2)^{\prime}$ There exists a function $m \in L^{1 / \beta}\left(I ; \mathbb{R}^{+}\right)$where $0<\beta<q$ and a nondecreasing continuous function $\Phi: \mathbb{R}^{+} \rightarrow \mathbb{R}^{+}$such that

$$
\|f(t, x)\| \leqslant m(t) \Phi(\|x\|)
$$

for all $x \in E$ and almost all $t \in I$. 
(H5)' There exists at least one solution $p \in C(I,(0, \infty))$ to the inequality

$$
\frac{\Phi\left(\|p\|_{0}\right)}{\Gamma(q)} \int_{0}^{t}(t-s)^{q-1} m(s) d s \leq p(t), \quad t \in I,
$$

where $\|\cdot\|_{0}$ is the sup norm in $C(I,(0, \infty))$.

Then equation (3.5) has at least one solution $u \in C(I, E)$.

Proof. Let $a(t)=t^{q-1} / \Gamma(q)$ in Theorem 3.1. From $(\mathrm{H} 2)^{\prime}$ we have $0<\beta<q<1$ for some $\beta$. It follows that

$$
\begin{aligned}
& \frac{1}{\Gamma(q)^{1 /(1-\beta)}} \int_{0}^{t}\left[(\tau+h)^{q-1}-\tau^{q-1}\right]^{1 / 1-\beta} d \tau \\
& \leq \frac{1}{\Gamma(q)^{1 /(1-\beta)}} \int_{0}^{t}\left[(\tau+h)^{(q-1) /(1-\beta)}-\tau^{(q-1) /(1-\beta)}\right] d \tau \\
&= \frac{1}{\Gamma(q)^{1 /(1-\beta)}} \frac{1-\beta}{q-\beta}\left[(t+h)^{(q-\beta) /(1-\beta)}-h^{(q-\beta) /(1-\beta)}\right. \\
&\left.-t^{(q-\beta) /(1-\beta)}\right] \rightarrow 0,
\end{aligned}
$$

as $h \rightarrow 0$, where we have used the inequality $(a-b)^{r} \leq a^{r}-b^{r}$, $a>b>0, r>0$. This shows (H3).

On the other hand, note that $(\mathrm{H} 5)$ is a direct consequence of $(\mathrm{H} 5)^{\prime}$. The conclusion follows from Theorem 3.1.

Remark 3.4. Note that the Hausdorff measure of noncompactness of the unit ball in finite-dimensional $E=\mathbb{R}^{n}$ is zero. It follows that condition (H3) is redundant. Thus, we obtain the following result.

Corollary 3.5. Suppose that conditions $(\mathrm{H} 1)-(\mathrm{H} 2)$ and $(\mathrm{H} 4)-(\mathrm{H} 5)$ are satisfied. Then the following system of nonlinear Volterra integral equations has at least one solution, $u \in C\left(I, \mathbb{R}^{n}\right)$,

$$
u(t)=\int_{0}^{t} a(t-s) f(s, u(s)) d s \quad t \in[0, T],
$$

where $u$ is a vector-valued function. 
4. An application. In this section, we present one example which does not aim at generality but indicates how our theorems can be applied to more concrete problems.

Example 4.1. Let $c_{0}$ the space of all sequences converging to zero. We know that $c_{0}$ is a Banach space with the norm:

$$
\|x\|_{\infty}=\sup _{k \in \mathbb{Z}}\left|x_{k}\right| \text {. }
$$

Consider the following Volterra equation:

$$
u(t)=\int_{0}^{t} e^{-\delta(t-s)} f(s, u(s)) d s, \quad t \in I:=[0, T],
$$

where $0<\delta<2,0<T<1 / 2$ and $f: I \times c_{0} \rightarrow c_{0}$ is given by

$$
\begin{gathered}
f(t, x)=\frac{1}{t^{2}+1 / 2}\left(\ln \left(\left|x_{k}\right|+1\right)+\frac{t}{k^{2}}\right)_{k=1}^{\infty}, \\
t \in I, \quad x=\left(x_{k}\right)_{k} \in c_{0} .
\end{gathered}
$$

We prove the existence of a solution $u \in C\left(I, c_{0}\right)$ for equation (4.1). To do this, we verify conditions (H1)-(H5).

(a) For every $x \in c_{0}$, we have that the function

$$
t \longmapsto f(t, x)=\frac{1}{t^{2}+1 / 2}\left(\ln \left(\left|x_{k}\right|+1\right)+\frac{t}{k^{2}}\right)_{k=1}^{\infty}
$$

is Lebesgue measurable since it is a continuous function for all $t \geq 0$.

(b) Let $t \in I$ be given. We will see that the function

$$
x \longmapsto f(t, x)
$$

is continuous on $c_{0}$. Recall that earlier we showed that the mean value theorem implies that the function $g(s)=\ln (s+1)$ (for $s>0$ ) is uniformly continuous on $(0, \infty)$. Now, let $\left(x^{n}\right)$ be a sequence in $c_{0}$ such that $x^{n} \rightarrow x$ in $c_{0}$ as $n \rightarrow \infty$. Then

$$
\begin{aligned}
& \sup _{k}\left|f\left(t, x_{k}^{n}\right)-f\left(t, x_{k}\right)\right| \\
& \left.\leq \frac{1}{t^{2}+1 / 2} \sup _{k} \mid \ln \left(\left|x_{k}^{n}\right|+1\right)-\ln \left(\left|x_{k}\right|+1\right)\right) \mid
\end{aligned}
$$




$$
\begin{aligned}
& \leq \frac{1}{t^{2}+1 / 2} \sup _{k}|| x_{k}^{n}|-| x_{k}|| \\
& \leq \frac{1}{t^{2}+1 / 2} \sup _{k}\left|x_{k}^{n}-x_{k}\right| \longrightarrow 0, \quad n \rightarrow \infty, t \in I .
\end{aligned}
$$

Hence, $\left\|f\left(t, x^{n}\right)-f(t, x)\right\|_{\infty} \rightarrow 0$ as $n \rightarrow \infty$ for all $t \in I$. Thus, we conclude that $f(t, \cdot)$ is continuous for all $t \in I$.

(H2) We define $m: I \rightarrow \mathbb{R}^{+}$and $\Phi: \mathbb{R}^{+} \rightarrow \mathbb{R}^{+}$by

$$
m(t)=\frac{1}{t^{2}+1 / 2} \quad \text { and } \quad \Phi(t)=t+T .
$$

Let $t \in I$ and $x=\left(x_{k}\right)_{k}$ in $c_{0}$ be given. Then, we have

$$
\begin{aligned}
\|f(t, x)\|_{\infty} & =\left\|\frac{1}{t^{2}+1 / 2}\left(\ln \left(\left|x_{k}\right|+1\right)+\frac{t}{k^{2}}\right)_{k}\right\|_{\infty} \\
& \leq \frac{1}{t^{2}+1 / 2}\left(\sup _{k}\left|x_{k}\right|+t\right) \\
& \leq \frac{1}{t^{2}+1 / 2}\left(\|x\|_{\infty}+T\right)=m(t) \Phi\left(\|x\|_{\infty}\right) .
\end{aligned}
$$

Hence, (H2) holds.

(H3) We define $H: I \rightarrow \mathbb{R}^{+}$and $\phi: \mathbb{R}^{+} \rightarrow \mathbb{R}^{+}$by

$$
H(t)=\frac{1}{t^{2}+1 / 2} \quad \text { and } \quad \phi(t)=\ln (t+1) .
$$

Note that $H \in L^{1}\left(I ; \mathbb{R}^{+}\right)$. Also, we can observe that $\phi$ is an increasing function and $\lim _{n \rightarrow \infty} \phi^{n}(t)=0$ (see [1, Lemma 2.1 and Remark 3.2]). We will prove that, for any bounded set $B \subset E$,

$$
\gamma(f(t, B)) \leq H(t) \phi(\gamma(B)) .
$$

Indeed, we recall that the Hausdorff measure of noncompactness $\gamma$ in the space $c_{0}$ can be computed by means of the formula:

$$
\gamma(B)=\lim _{n \rightarrow \infty} \sup _{x \in B}\left\|\left(I-P_{n}\right) x\right\|_{\infty},
$$

where $B$ is a bounded subset of $c_{0}$ and $P_{n}$ is the projection onto $V_{n}=\operatorname{span}\left\{e_{1}, \ldots, e_{n}\right\}$ of the first $n$ vectors in the standard basis (see [4]). Next, let $u=\left(u_{k}\right)_{k} \in B \subset c_{0}$ and $t \in I$. Fix $n \in \mathbb{N}$. Thus, using 
the fact that $P_{n}$ is the identity operator on $V_{n}$, we obtain

$$
\ln \left(\left|u_{k}\right|+1\right) \leq \ln \left(\left\|\left(I-P_{n}\right)\left(u_{k}\right)_{k}\right\|_{\infty}+1\right) \quad \text { for all } k>n .
$$

Then

$$
\begin{aligned}
\sup _{u \in B}\left\|\left(I-P_{n}\right)\left(\ln \left(\left|u_{k}\right|+1\right)_{k}\right)\right\|_{\infty} & =\sup _{u \in B} \sup _{k>n}\left|\ln \left(\left|u_{k}\right|+1\right)\right| \\
\leq & \sup _{u \in B} \ln \left(\left\|\left(I-P_{n}\right)\left(u_{k}\right)_{k}\right\|_{\infty}+1\right) \\
& =\ln \left(\sup _{u \in B}\left\|\left(I-P_{n}\right)\left(u_{k}\right)_{k}\right\|_{\infty}+1\right) .
\end{aligned}
$$

Letting $n \rightarrow \infty$, we get

$$
\begin{aligned}
& \lim _{n \rightarrow \infty} \sup _{u \in B}\left\|\left(I-P_{n}\right)\left(\ln \left(\left|u_{k}\right|+1\right)_{k}\right)\right\|_{\infty} \\
& =\sup _{u \in B} \sup _{k>n}\left|\ln \left(\left|u_{k}\right|+1\right)\right| \\
& \leq \lim _{n \rightarrow \infty} \ln \left(\sup _{u \in B}\left\|\left(I-P_{n}\right)\left(u_{k}\right)_{k}\right\|_{\infty}+1\right) \\
& =\ln \left(\lim _{n \rightarrow \infty} \sup _{u \in B}\left(\left\|\left(I-P_{n}\right)\left(u_{k}\right)_{k}\right\|_{\infty}\right)+1\right) .
\end{aligned}
$$

On the other hand, for $k>n$, we have that

$$
\left(I-P_{n}\right)\left(\frac{t}{k^{2}}\right)_{k}=\left(\frac{t}{k^{2}}\right)_{k} \leq\left(\frac{t}{n^{2}}, \frac{t}{n^{2}}, \ldots\right),
$$

and consequently,

$$
\lim _{n \rightarrow \infty} \sup _{u \in B}\left\|\left(I-P_{n}\right)\left(\frac{t}{k^{2}}\right)_{k}\right\|_{\infty}=0 .
$$

It follows that

$$
\begin{aligned}
\gamma(f(t, B)) & =\frac{1}{t^{2}+1 / 2} \lim _{n \rightarrow \infty} \sup _{u \in B}\left\|\left(I-P_{n}\right)\left(\ln \left(\left|u_{k}\right|+1\right)+\frac{t}{k^{2}}\right)_{k}\right\|_{\infty} \\
& \leq \frac{1}{t^{2}+1 / 2} \lim _{n \rightarrow \infty} \sup _{u \in B}\left\|\left(I-P_{n}\right)\left(\ln \left(\left|u_{k}\right|+1\right)_{k}\right)\right\|_{\infty} \\
& \leq \frac{1}{t^{2}+1 / 2} \ln \left(\lim _{n \rightarrow \infty} \sup _{u \in B}\left(\left\|\left(I-P_{n}\right)\left(u_{k}\right)_{k}\right\|_{\infty}\right)+1\right) \\
& =H(t) \phi(\gamma(B)) .
\end{aligned}
$$

This proves (H5) is clear from the claim, and hence condition (H3) is satisfied. 
(H4) is clear from the continuity of $a(t)$.

(H5) We see that there is a solution $p(t) \in C\left(I, \mathbb{R}^{+}\right)$to the equation

$$
\Phi\left(\|p\|_{0}\right) \int_{0}^{t} a(t-s) m(s) d s \leq p(t), \quad t \in I .
$$

Note that

$$
(1 * a)(T)=\int_{0}^{T} a(s) d s=\int_{0}^{T} e^{-\delta s} d s=\frac{1}{\delta}\left(1-e^{-\delta T}\right) .
$$

Now, we can see (using the mean value theorem) that $1 / 2 \leq(1 / \delta) \ln (2 /$ $(2-\delta))$ for $0<\delta<2$. Since $0<T<1 / 2$ we get that $0<T<$ $(1 / \delta) \ln (2 /(2-\delta))$. Then

$$
0<(1 * a)(T)<\frac{1}{2} .
$$

Let $p(t)=\lambda$, where

$$
\lambda \geq \frac{2 T(1 * a)(T)}{1-2(1 * a)(T)} .
$$

Then

$$
\begin{aligned}
\Phi\left(\|p\|_{0}\right) \int_{0}^{t} a(t-s) m(s) d s & =\Phi(\lambda) \int_{0}^{t} \frac{e^{-\delta s}}{s^{2}+1 / 2} d s \\
& \leq 2(\lambda+T) \int_{0}^{T} e^{-\delta s} d s \\
& =2(\lambda+T)(1 * a)(T) \leq \lambda .
\end{aligned}
$$

By Theorem 3.1, we obtain the existence of at least one solution to the Volterra equation (4.1).

Remark 4.2. If we consider any kernel $a(t)$ such that (H4) holds and by taking $p(t)=\lambda$ such that the inequality (4.3) holds, then the problem (4.1) always has at least one solution for a suitable value of $T$, more precisely, for $T$ satisfying:

$$
0<(1 * a)(T)<\frac{1}{2} .
$$


Remark 4.3. We observe that Volterra equation (4.1) in the Banach space $E=c_{0}$ is equivalent to the nonlinear lattice model:

$$
\left\{\begin{array}{l}
u_{t}(t, n)+\delta u(t, n)=f(t, u(t, n)) \quad t \in[0, T], \quad n \in \mathbb{Z}, \\
u(0, n)=0
\end{array}\right.
$$

Consequently, the above example says that, for the given nonlinear term, and whenever $0<T<1 / 2$ and $0<\delta<2$, there exists a solution satisfying $|u(t, n)| \rightarrow 0$ as $n \rightarrow \infty$.

\section{REFERENCES}

1. A. Aghajani, J. Banaś and N. Sabzali, Some generalizations of Darbo fixed point theorem and applications, Bull. Belgian Math. Soc. 20 (2013), 345-358.

2. A. Aghajani and E. Pourhadi, Application of measure of noncompactness to $l_{1}$-solvavility of infinite system of second order differential equations, Bull. Belgian Math. Soc. 22 (1) (2015), 105-118.

3. A. Aghajani, E. Pourhadi and J.J. Trujillo, Application of measure of noncompactness to a Cauchy problem for fractional differential equations in Banach spaces, Fract. Calc. Appl. Anal. 16 (2013), 962-977.

4. R.R. Akhmerov, M.I. Kamenskii, A.S. Potapov, A.E. Rodkina and B.N. Sadovskii, Measure of noncompactness and condensing operators, Birkhäuser Verlag, Basel, 1992.

5. R. Allahyari, R. Arab and H. Shole Haghighi, Existence of solutions for some classes of integro-differential equations via measure of noncompactness, Electr. J. Qual. Theor. Differ. Equat. 41 (2015), 1-18.

6. E. Alvarez and C. Lizama, Attractivity for functional Volterra integral equations of convolution type, J. Comp. Appl. Math. 30 (2016), 230-240.

7. M.R. Arias, R. Benitez and V.J. Bolós, Nonconvolution nonlinear integral Volterra equations with monotone operators, Comp. Math. Appl. 50 (2005), 14051414.

8. J. Banaś and K. Goebel, Measure of noncompactness in Banach spaces, Lectures Notes Pure Appl. Math. 60, Dekker, New York, 1980.

9. J. Banaś and M. Mursaleen, Sequence spaces and measures of noncompactness with applications to differential and integral equations, Springer, New Delhi, 2014.

10. J. Banaś and R. Nalepa, On a measure of noncompactness in the space of functions with tempered increments, J. Math. Anal. Appl. 435 (2016), 1634-1651.

11. D. Bothe, Multivalued pertubations of $m$-accretive differential inclusions, Israel. J. Math. 108 (1998), 109-138.

12. G. Gripenberg, S.-O. Londen and O. Staffans, Volterra integral and functional equations, in Encyclopedia of mathematics and its applications, 34, Cambridge University Press, Cambridge, 1990. 
13. F.R. de Hoog and R.S. Anderssen, Kernel perturbations for a class of secondkind convolution Volterra equations with non-negative kernels, Appl. Math. Lett. 25 (2012), 1222-1225.

14. M. Kazemi and R. Ezzati, Existence of solution for some nonlinear twodimensional Volterra integral equations via measures of noncompactness, Appl. Math. Comp. 275 (2016), 165-171.

15. H. Khosravi, R. Allahyari and A. Shole Haghighi, Existence of solutions of functional integral equations of convolution type using a new construction of a measure of noncompactness on $L_{p}\left(\mathbb{R}_{+}\right)$, Appl. Math. Comp. 260 (2015), 140-147.

16. C. Lizama and M.P. Velasco, Abstract Volterra equations with statedependent delay, J. Integral Equat. Appl. 27 (2015), 219-231.

17. F. Mainardi, Fractional calculus and waves in linear viscoleasticity. An introduction to mathematical models, Imperial College Press, London, 2010.

18. A. Mallika Arjunan, V. Kavitha and S. Selvi, Existence results for impulsive differential equations with nonlocal conditions via measures of noncompactness, J. Nonlin. Sci. Appl. 5 (2012), 195-205.

19. M. Mursaleen and S.A. Mohiuddine, Applications of measure of noncompactness to the infinity system of differential equations in $\ell_{p}$ spaces, Nonlin. Anal. 75 (2012), 2111-2115.

20. M. Mursaleen and S.M.H. Rizvi, Solvability of infinite system of second order differential equations in $c_{0}$ and $\ell_{1}$ by Meir-Keeler condensing operator, Proc. Amer. Math. Soc., doi:10.1090/proc/13048.

21. J.R. Wang, Y. Zhou and M. Fečkan, Abstract Cauchy problem for fractional differential equations, Nonlin. Dynam. 71 (2013), 685-700.

Universidad del Norte, Departamento de Matemáticas y Estadística, BarRANQUilla, AA 1569, Colombia

Email address: edgalp@yahoo.com, ealvareze@uninorte.edu.co

Universidad de Santiago de Chile, Facultad de Ciencia, Departamento de Matemática y Ciencia de la Computación, Casilla 307, Correo 2, Santiago, CHiLe

Email address: carlos.lizama@usach.cl 